\title{
ORIGINAL ARTICLE Closing the gaps: Steps towards elimination of mother-to-child transmission of HIV
}

M Ibeto, ${ }^{1}$ BSc, MB ChB; J Giddy, ${ }^{2} \mathrm{MB}$ ChB, MFamMed; V Cox, ${ }^{1} \mathrm{MD}$

${ }^{1}$ Médecins Sans Frontières, Khayelitsha, Cape Town, South Africa

${ }^{2}$ Western Cape Department of Health, Khayelitsha and Eastern Substructure, Cape Town, South Africa

Corresponding author: M Ibeto (msfocb-khayelitsha-doc2@brussels.msf.org)

Background. With significant reductions in the rate of HIV mother-to-child transmission (MTCT) in South Africa, each case of failed prevention of MTCT (PMTCT) should be investigated.

Objective. To establish the cause(s) of MTCT at Khayelitsha's Community Health Centre (CHC) in order to identify obstacles to MTCT elimination. Methods. Routinely collected data were reviewed for all HIV-infected infants identified at Khayelitsha Site B CHC from January 2012 to April 2013. Results. A total of 926/1 158 (80\%) of exposed infants had polymerase chain reaction (PCR) results, with 15/926 (1.6\%) PCR-positive. Median (interquartile range (IQR)) values for the maternal indicators were as follows: maternal age, 27 (23 - 31) years; parity, 2 (1 - 3); gestational age at

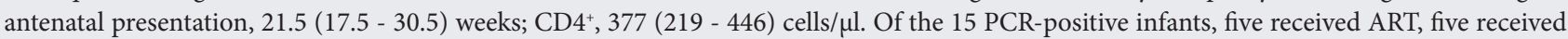
AZT and five received no prophylaxis. Viral loads were not monitored for any of the women receiving antenatal ART. Nine of the 15 (60\%) delivered in hospital, with 6/9 requiring caesarean section. The median (IQR) infant birth weight was 3.0 (2.6 - 3.5) kg. All received prophylactic nevirapine post exposure. Two of the 15 were clinically unwell at birth, and 14 (86.7\%) were breastfed, with 10 (66.7\%) recorded as exclusively breastfed. Median (IQR) time between delivery and PCR results was 6.6 (6.1 - 7.3) weeks.

Discussion. PMTCT programmes must consider each PCR-positive infant as a sentinel event that can provide valuable insight into correcting ongoing clinical and programmatic reasons for HIV transmission. The main risk factors for MTCT identified in this study were late presentation for antenatal care, inadequate antenatal PMTCT prophylaxis and a lack of viral load monitoring.

S Afr J HIV Med 2014;15(3):107-109. DOI:10.7196/SAJHIVMED.1047 
Over the last decade, South Africa (SA) has experienced dramatic improvements in its prevention of mother-to-childtransmission (PMTCT) programme. The first national-level PMTCT programme was introduced in 2002 and included single-dose nevirapine (Sd-NVP) during labour for the mother and to the baby postpartum, modified obstetric practices, infant feeding counselling, and the provision of free infant formula to HIV-positive mothers who chose not to breastfeed. ${ }^{[1]}$ In 2005, pregnant women with $\mathrm{CD} 4^{+}$counts $\leq 200$ cells $/ \mu$ l were eligible for triple-drug antiretroviral (ARV) therapy (ART) for their own health, and in 2008 pregnant women with $\mathrm{CD}^{+}$counts $>200$ cells/ $\mu$ l were offered 'dual therapy' (azidothymidine (AZT) from 28 weeks, and Sd-NVP in labour) and SdNVP with AZT postnatal infant prophylaxis. In 2010, there were further improvements to the PMTCT programme. These included routine HIV testing and counselling for pregnant women, dual therapy to prevent MTCT of HIV, highly active ARV therapy for pregnant women with a $\mathrm{CD} 4^{+}$cell count $\leq 350$ cells/ $\mu$ l, postnatal infant prophylaxis with NVP for breastfeeding HIV-positive women and intensified efforts to integrate PMTCT services into routine maternal and child health $(\mathrm{MCH})$ services. ${ }^{[2]}$ The success of the national PMTCT programme was seen in the 2010 SA PMTCT evaluation (the first national evaluation of the PMTCT programme in SA), which demonstrated a significant reduction in MTCT to 3.5\% (compared with an estimated transmission risk of $20-30 \%$ without any intervention). ${ }^{[3]}$ The SA PMTCT evaluation study also showed high uptake of PMTCT services nationally, with more than 98\% of women getting an HIV test during pregnancy and $92 \%$ of HIV-infected mothers receiving ARV treatment or prophylaxis. ${ }^{[3]}$ SA's latest and arguably most controversial PMTCT improvements came in April 2013: SA followed the World Health Organization recommendations by offering ART to all HIVpositive pregnant or breastfeeding women regardless of $\mathrm{CD}^{+}$count. $^{[4]}$ In light of such achievements, what more is needed to 'close the gaps' in the PMTCT cascade and reach the Millennium Development Goal of elimination of MTCT by 2015?

The objective of this review was to establish possible cause(s) of transmission for HIVinfected infants in order to identify ongoing obstacles to elimination of MTCT. Antenatal and PMTCT history, delivery information and feeding options were obtained from routinely collected data held in antenatal clinic registers and clinical files.

\section{Methods}

For this case series, Médecins Sans Frontières (MSF) partnered with the Western Cape Department of Health (WC DoH) to review and document case histories of all PCR-positive infants found in the HIV-exposed infant register at Khayelitsha's Site B Community Health Centre in Cape Town from 1 January 2012 to 30 April 2013. Khayelitsha’s Site B Community Health Centre is a campus that provides primary healthcare services including casualty, outpatient department for adults and children, an HIV/tuberculosis (TB)/drug-resistant TB (DR-TB) clinic, and a midwifery and obstetrics unit. Within its paediatric services, it has a baby clinic that is dedicated to the registration and care of HIV-exposed infants. It is the responsibility of this baby clinic to ensure that all HIV-exposed infants who attend receive a PCR test at around 6 weeks postpartum.

\section{Results}

A total of 1158 HIV-exposed infants were recorded in the exposed infant register at Khayelitsha Site B Community Health Centre over the 16-month study period (from 1 January 2012 to 30 April 2013). Of these, 926 (80\%) of exposed infants had HIV DNA PCR results available, and 15 (1.6\%) were PCR-positive.

Review of the maternal histories for the 15 positive infants (Table 1) found that the median (interquartile range (IQR)) maternal age was $27(23-31)$ years, median parity was $2(1-3)$, and median gestational age at antenatal presentation was $21.5(17.5-30.5)$ weeks. Baseline $\mathrm{CD} 4^{+}$count was $<350$ cells $/ \mu \mathrm{l}$ in $46.7 \%$ of women, with a median (IQR) $\mathrm{CD}^{+}$count of $377(219-446)$ cells/ $\mu$ l. Onethird of these mothers received ART: two started ART after 33 weeks' gestation and received no more than 5 weeks of ART prior to delivery, one started ART at 17 weeks' gestation but then defaulted treatment at month 1 on ART, and the remaining two started ART at 25 and 21 weeks' gestation, respectively, but had clinically unwell babies at birth, suggesting early in utero transmission. Five mothers received AZT for a median duration of 20 weeks (based on clinical records only; no record of degree of adherence to AZT dual therapy was found) and an additional five received no PMTCT prophylaxis (two defaulted on ART prior to

\begin{tabular}{|c|c|}
\hline \multicolumn{2}{|l|}{ Patient characteristics $(N=15)$} \\
\hline Maternal age (years), median (IQR) & $27(23-31)$ \\
\hline Parity, median (IQR) & $2(1-3)$ \\
\hline Gestation at 1st ANC presentation (weeks), median (IQR) & $21.5(17.5-30.5)$ \\
\hline Maternal CD4 ${ }^{+}$count (cells/ $\left.\mu \mathrm{l}\right)$, median (IQR) & $377(219-446)$ \\
\hline \multicolumn{2}{|l|}{$\mathrm{CD} 4^{+}$count $($cells/ $\mu \mathrm{l}), n(\%)$} \\
\hline$<200$ & $3(20.0)$ \\
\hline $200-349$ & $4(26.7)$ \\
\hline$\geq 350$ & $8(53.3)$ \\
\hline \multicolumn{2}{|l|}{ PMTCT prophylaxis provided, $n(\%)$} \\
\hline ART & $5(33.3)$ \\
\hline $\mathrm{AZT}$ & $5(33.3)$ \\
\hline No prophylaxis & $5(33.3)$ \\
\hline Mothers requiring hospital care at delivery, $n(\%)$ & $9(60)$ \\
\hline Infant birth weight $(\mathrm{kg})$, median (IQR) & $3.0(2.6-3.5)$ \\
\hline Infants clinically unwell at birth, $n(\%)$ & $2(13.3)$ \\
\hline \multicolumn{2}{|l|}{ Breastfed infants, $n(\%)$} \\
\hline Documented breastfeeding of any duration & $14(86.7)$ \\
\hline Documented exclusive breastfeeding & $10(66.7)$ \\
\hline Duration between delivery and PCR test (weeks), median (IQR) & $6.6(6.1-7.3)$ \\
\hline
\end{tabular}


onset of pregnancy but verbally reported being on ART at booking, one presented in labour, one tested negative in pregnancy then positive in labour, and one did not receive prophylaxis despite booking at 6 weeks' gestation). Viral loads were not monitored for women on antenatal ART, as the national ART/PMTCT guidelines at the time required 1st viral load to be drawn at month 4 on ART and not prior. Out of 15 mothers, $9(60 \%)$ required hospital care at delivery, with 6 requiring caesarean section.

Review of the early infant histories for the 15 positive infants revealed the following: median (IQR) infant birth weight was $3.0(2.6-3.5) \mathrm{kg}$. all received nevirapine as postexposure prophylaxis (the duration and adherence to which was unclear from clinic records); and 14 (87\%) were breastfed with 10 (67\%) recorded as 'exclusively breastfed'. Median (IQR) time between delivery and PCR results was 6.6 (6.1 - 7.3) weeks.

\section{Discussion}

This case series points to several critical gaps in the PMTCT cascade in this setting. It was of great concern to discover that, consistently over the 16 -month period, only $80 \%$ of exposed infants had documented PCR results. This finding is of particular concern when considered in the context of the SA PMTCT evaluation study reported intention to obtain infant PCR testing at 6 weeks the study found that only $35 \%$ of HIV-positive mothers indicated that they planned to obtain early infant diagnosis for their infant during their 6-week immunisation visit. Multiple possible causes were identified for the suboptimal PCR testing rate, including poor patient education of the need to return at around 6 weeks for a PCR test, poor staff knowledge (because of staff turnover and shortages) of the need to ensure a PCR is done in infants presenting between 6 weeks and 18 months, the fragmentation of child health services within the facility, and insufficient staff motivation to follow up on missing PCR results or infants not returning for their initial PCR test. Further research is required to analyse in more detail all the causes of inadequate infant PCR testing coverage, along with intensified effort to improve the postnatal linkage to care of all HIV-exposed infants and their mothers.

Analysis of the maternal antenatal histories for infants found to be HIV-infected revealed further gaps in the PMTCT cascade. The need to intensify community awareness of the importance of early antenatal booking was highlighted in the following findings: median (IQR) gestational age at antenatal presentation was 21.5 (17.5 - 30.5) weeks, and one mother presented for the first time in labour (no antenatal care) despite this being her 3rd pregnancy and already knowing her HIV status. To address this gap, MSF and WC DoH have partnered with the Treatment Action Campaign to launch a community awareness campaign that aims to improve patient literacy particularly on the issue of early antenatal booking.

Inconsistency in the provision of antenatal prophylaxis and a lack of viral load monitoring for pregnant or breastfeeding women on ART were identified as further significant gaps. From clinical records, the median duration of AZT dual therapy was 20 weeks, but was complicated by: poor maternal understanding of the importance of adherence to AZT twice daily; pregnant women often running out of their AZT between appointments; staff not routinely checking AZT pill counts during antenatal visits; and no system for monitoring AZT provision or adherence. One mother, whose child is now HIV-infected, was not provided antenatal AZT prophylaxis despite booking at 6 weeks' gestation and presenting a further four times in her pregnancy; this woman was told she had booked too early to start prophylaxis on her first visit, and antenatal staff failed to detect that she had not been issued AZT on each subsequent visit. The absence of routine viral load monitoring for women on ART at antenatal booking and subsequently through pregnancy and breastfeeding resulted in failing to detect women who had defaulted ART prior to or during pregnancy; two mothers of HIVinfected infants in our review defaulted on ART prior to onset of pregnancy but verbally reported being on ART at their antenatal booking. Vigilance in viral load monitoring for women on ART during pregnancy is essential, and infant feeding choice should take into account a woman's actual or likely viral load at delivery. Of the 15 HIV-infected infants, 14 were breastfed, with 10 documented as being exclusively breastfed. In these cases, HIV transmission may have occurred due to breastfeeding in the presence of a high viral load. In a small number of cases, a complicated delivery was also linked with transmission.

These data come from a case series, and as such, have fundamental limitations related to absence of a comparator group of HIV-exposed but uninfected infants. Nonetheless, several key concerns emerge clearly. The main risk factors for MTCT identified in our review of the case histories of PCR-positive infants were late presentation to antenatal care, inadequate provision or duration of antenatal PMTCT prophylaxis, and a lack of viral load monitoring to determine whether a pregnant woman is virologically suppressed at delivery and during breastfeeding. Women with any of these risk factors should be urgently identified as high risk for transmission and provided additional adherence support, home visits by community care workers, an early infant PCR test, tailored infant feeding advice and dual postnatal infant prophylaxis.

Recent policy developments calling for provision of ART for all HIVpositive pregnant or breastfeeding women regardless of $\mathrm{CD} 4^{+}$count is welcomed, and will undoubtedly improve PMTCT outcomes in SA. However, the elimination of MTCT will require intensified efforts to strengthen each step in the PMTCT cascade, from promotion of early antenatal booking to timely identification and appropriate management of high-risk pregnant or breastfeeding women. The introduction of routine birth PCR testing for high-risk infants would allow for early detection of infants infected with HIV; this would reduce the risk that HIV-positive infants are lost to follow-up prior to diagnosis and allow rapid initiation of ART. PMTCT programmes must consider each PCR-positive infant as a sentinel event that can provide valuable insight into correcting ongoing clinical and programmatic reasons for HIV transmission.

\section{References}

1. National Department of Health South Africa. Policy and Guidelines for the Implementation of the PMTCT Programme. Pretoria: National Department of Health South Africa, 2001

2. National Department of Health South Africa/The South African National AIDS Council. Clinical Guidelines: PMTCT (Prevention of Mother-to-Child Transmission). Pretoria: National Department of Health South Africa and The South African National AIDS Council, 2010.

3. Goga AE, Dinh TH, Jackson DJ for the South African PMTCT Evaluation study group. Evaluation of the Effectiveness of the National Prevention of Mother-toChild Transmission (PMTCT) Programme Measured at Six Weeks Postpartum in South Africa, 2010. South African Medical Research Council, National Department of Health of South Africa and President's Emergency Plant for AIDS Relief (PEPFAR/US) Centers for Disease Control and Prevention, 2012.

4. National Department of Health South Africa. The South African Antiretroviral Treatment Guideline 2013, PMTCT Guidelines, Revised March 2013. Pretoria: National Department of Health South Africa, 2013. 